\title{
artigo
}

\section{Treino de força e estresse durante a pandemia do} COVID-19

\author{
Strength and stress training during the COVID-19 pandemic \\ Entrenamiento de fuerza y estrés durante la pandemia de COVID-19
}

\begin{abstract}
RESUMO
Objetivo avaliar o efeito de uma sessão de treino de força nos níveis de cortisol em homens e correlacionar ao score de estresse durante a pandemia do COVID-19. Participaram do estudo 10 voluntários fisicamente ativos com idade de 18 a 31 anos. Após repouso de 48 horas foi aplicado uma anamnese, avaliação da composição corporal, hemodinâmica em repouso, avaliação da variabilidade da frequência cardíaca, coleta de sangue. Foi observada redução nos níveis de cortisol um dia após a sessão de treino de força. Uma forte correlação foi observada entre o score de estresse e os níveis de cortisol bem como entre o cortisol e o índice LFms em repouso e entre a razão SD1/SD2 e o peso magro. Uma única sessão de treino de força com alta intensidade se mostrou eficaz na redução do cortisol em homens adultos que apresentaram níveis elevados de estresse percebido durante a pandemia do COVID-19.
\end{abstract}

DESCRITORES: Cortisol; Variabilidade da Frequência da Cardíaca; Massa Magra.

\section{ABSTRACT}

Objective: To evaluate the effect of a strength training session on men's cortisol levels and correlate to the stress score during the COVID-19 pandemic. Ten physically active volunteers aged 18 to 31 years participated in the study. After a 48-hour rest, an anamnesis, body composition assessment, hemodynamics at rest, assessment of heart rate variability, blood collection was applied. A reduction in cortisol levels was observed one day after the strength training session. A strong correlation was observed between the stress score and cortisol levels and between cortisol and the LFms index at rest, and between the SD1 / SD2 ratio and lean weight. A single session of high-intensity strength training effectively reduced cortisol in adult men who had high levels of perceived stress during the COVID-19 pandemic.

DESCRIPTORS: Cortisol; Heart Rate Variability; Lean Mass.

\section{RESUMEN}

Objetivo evaluar el efecto de una sesión de entrenamiento de fuerza sobre los niveles de cortisol en hombres y correlacionarlo con la puntuación de estrés durante la pandemia de COVID-19. Participaron en el estudio diez voluntarios físicamente activos de entre 18 y 31 años. Después de un descanso de 48 horas, se aplicó una anamnesis, valoración de la composición corporal, hemodinámica en reposo, valoración de la variabilidad de la frecuencia cardíaca, extracción de sangre. Se observó una reducción en los niveles de cortisol un día después de la sesión de entrenamiento de fuerza. Se observó una fuerte correlación entre la puntuación de estrés y los niveles de cortisol, así como entre el cortisol y el índice LFms en reposo y entre la relación SD1 / SD2 y el peso magro. Una sola sesión de entrenamiento de fuerza de alta intensidad fue efectiva para reducir el cortisol en hombres adultos que tenían altos niveles de estrés percibido durante la pandemia de COVID-19.

DESCRIPTORES: Cortisol; Variabilidad del ritmo cardíaco; Masa magra.

RECEBIDO EM: 13/01/2021 APROVADO EM: 05/02/2021

\section{José Morais Souto Filho}

Mestre em Educação Física Pela Universidade Católica de Brasília - DF, Especialização em Educação Física Escolar pela Faculdade Integrada de Patos-PB, Graduado em Licenciatura Pena em Educação Física pela Autarquia de Ensino Superior de Arcoverde -PE, Integrante do grupo de estudos do desempenho humano e das repostas fisiológicas ao exercício, Professor efetivo no curso de bacharelado em Educação Física da Faculdade de Integração do Sertão.

ORCID: 0000-0001-8874-1708 


\section{Karlla Giselle Coelho Farias da Silva}

Bacharel e Licenciada em Educação Física pela Universidade de Pernambuco. ORCID: 0000-0002-6763-0248

\section{Ivo Eduardo Galvão Modesto}

Licenciado em Educação Física pela Faculdade Montenegro, Pós Graduado pela Universidade Regional do Cariri. ORCID: 0000-0001-5450-240X

\section{INTRODUÇÃO}

0 coronavírus (COVID) teve seu primeiro caso relatado de infecção humana em Wuhan na China em $1^{\circ}$ de dezembro de 2019. Refere-se a um grupo viral já conhecido na comunidade científica desde a década de $1960^{1,2}$. A COVID-19 é uma doença altamente contagiosa que pode levar o paciente a desenvolver a Síndrome Respiratória Aguda Grave (SRAG). Até o início de janeiro de 2021 a COVID-19 já tinha vitimado mais de 1.930 .265 pessoas e infectado $89.048 .345 \mathrm{em}$ todo mundo. A magnitude da disseminação em praticamente todos os países do mundo, elevou em tempo recorde o status da COVID-19 como a maior pandemia do século XXI ${ }^{2}$. Na tentativa de evitar o colapso no sistema de saúde, diversos países adotaram a política de distanciamento social recomendado pela Organização Mundial da Saúde ${ }^{3,4}$. Tal medida extremamente necessária para conter o avanço devastador da doença. Ainda o medo da infecção, a perda de parentes e amigos, os impactos econômicos, dentre outros, provocou na população um alto nível de estresse, desencadeando diversos casos de síndrome do pânico, ansiedade ${ }^{5-7}$. O sedentarismo também cresceu consideravelmente em decorrência das restrições e fechamento dos espaços públicos e privados para a prática de atividades físicas. Este fato ocasionou o aumento da gordura corporal deixando a população mais predisposta ao desenvolvimento de doenças crônicas e metabólicas, evidenciando ainda mais os efeitos deletérios da pandemia da COVID-19 na saúde emocional e física da população mundial ${ }^{8,9}$. A exemplo: estudos vêm demostrando que indivíduos com sobrepeso e obesos apresentam maiores riscos de infecção respiratória grave quando infectados pelo COVID-19 ${ }^{10}$.
Sendo o exercício físico amplamente reconhecido como importante estratégia não medicamentosa na prevenção $\mathrm{e}$ no combate das doenças crônicas e metabólicas, no controle da massa corporal, é de fundamental importância no sistema imunológico. É sugerido que a prática de exercícios físicos se faça presente na rotina da população de forma segura e respeitando o devido distanciamento social durante a pandemia ${ }^{11}$. Entretanto, os efeitos do treinamento de força na atenuação do estresse provocado pela pandemia ainda não foram investigados.

Deste modo, o presente estudo teve como objetivo avaliar o efeito de um dia de treinamento de força nos níveis de cortisol em adultos e correlacionar ao score de estresse percebido durante o período da pandemia do COVID-19.

\section{MÉTODO}

Trata-se de um estudo de campo de caráter experimental exploratório, que se caracteriza pela busca da constatação de um fenômeno pouco investigado sobre o levantamento de hipótese prévia ${ }^{12}$. O estudo foi composto por 10 voluntários do sexo masculino saudáveis e fisicamente ativos com idade de 18 a 31 anos residentes na cidade de Triunfo, Pernambuco. A pesquisa foi realizada em setembro de 2020 e até a data das coletas nenhum participante do estudo tinha sido diagnosticado com a

\section{Figura 1. Desenho do Estudo. Autoria local 2020}

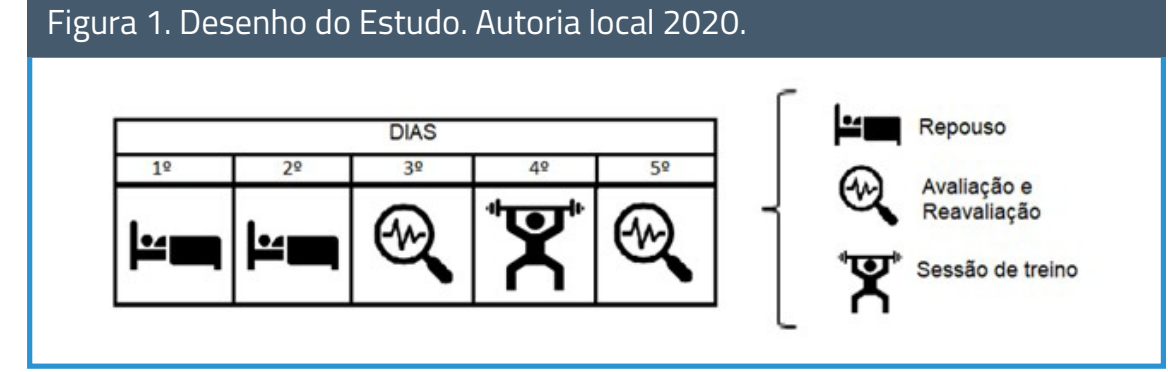

COVID-19. Para participação no estudo foram estabelecidos os seguintes critérios de inclusão: Não apresentar quaisquer doenças crônicas metabólicas ou transtornos emocionais diagnosticados, não estar fazendo uso de qualquer tipo de medicamentos ou suplementação e estar familiarizado com o treinamento de força. Os voluntários assinaram o Termo de Consentimento Livre Esclarecido de acordo com a Declaração de Helsinque. O projeto foi aprovado pelo Comitê de Ética em Pesquisa em Seres Humanos da Universidade Católica de Brasília, sob o protocolo número CAAE: 08754219.3.0000.0029.

Os voluntários em repouso de 48 horas foram submetidos a uma anamnese, avaliação da composição corporal, aferição da pressão arterial e frequência cardíaca em repouso de 15 minutos, avaliação da variabilidade da frequência cardíaca, coleta de sangue venoso para posterior análise da concentração de cortisol. Estes procedimentos foram realizados antes e um dia após a realização do treinamento de força.

A anamnese conteve questões sobre o histórico de saúde pessoal, nível de atividade física e nível de estresse percebido por meio da Perceived Stress Scale (PSS) traduzido. O questionário foi aplicado em forma de entrevista pelo avaliador principal. $\mathrm{O}$ PSS é um questionário de 14 questôes com opção de resposta de zero a quatro (zero = nunca; um = quase nunca; dois = as vezes; três $=$ quase sempre e quatro $=$ sempre). As 


\section{artigo}

perguntas positivas $(4,5,6,7,9,10$ e 13) deve ter sua pontuação somada de forma invertida. As demais somam-se de forma direta. O score é a soma da pontuação de cada pergunta, podendo variar de zero a cinquenta e seis. Quanto maior o escore maior será o estresse percebido ${ }^{13}$.

A estatura dos voluntários foi obtida utilizando um estadiômetro de parede, estando os avaliados com os pés, nádegas, escapulas e a porção posterior do crânio tocando a parede e a leitura realizada no momento de uma respiração profunda. Para a obtenção da massa corporal utilizou-se uma balança mecânica Filizola com precisão de 100 gramas. $\mathrm{O}$ índice de massa corporal (IMC) foi obtido por meio da equação: peso/(altura $)^{2}$. As medidas de circunferências (cintura e quadril) foram realizadas utilizando uma fita métrica flexível não elástica com escala em centímetros. Foi extraído a Relação Cintura Quadril (RCQ) dividindo a circunferências de cintura pelo quadril em centímetros. O percentual de gordura corporal foi obtido utilizando o protocolo de 3 dobras cutâneas ${ }^{14}$ com o auxílio de um adipômetro científico Cescof ${ }^{\circ}$. Após a determinação da densidade corporal pela equação $\mathrm{DC}=1,10938-0,0008267 \times$ (soma das dobras) $+0,0000016 x$ (soma das dobras $x$ soma das dobras) $-0,0002574 x$ (idade) 15 foi estimado o massa gordo (PG) pela seguinte equação: $P G=$ massa total $x$ (percentual de gordura/100) e massa magro $(\mathrm{PM})$ pela equação: $\mathrm{PM}=$ massa total - massa gordo. O percentual de gordura corporal por sua vez foi estimado pela equação de Siri, Percentual de Gordura= $495 / D C)-450^{14}$.

A pressão arterial e a frequência cardíaca foram obtidas após 15 minutos de repouso com a utilização de um aparelho eletrônico Microlife e um cardiofrequencímetro. A variabilidade da frequência cardíaca foi registrada durante 15 minutos utilizando um cardiofrequencímetro da marca Polar modelo RS800CX. Os voluntários foram orientados a não ingerirem café ou qualquer outro estimulante durante as 24 horas que antecederam a avaliação. Posteriormente os dados foram transferidos para o Softwere da Polar.
Para a remoção dos artefatos foi aplicado o filtro médio ${ }^{16}$. Os dados então foram analisados posteriormente utilizando o Softwere Kubios onde foram extraídos os dados no domínio do tempo e da frequência.

Cada participante foi colocado confortavelmente sentado com um torniquete amarrado em seu braço e uma coleta de 5 $\mathrm{ml}$ de sangue foi realizada na veia cubital mediana após assepsia do local, usando agulhas estéreis em tubos de soro. Nenhum dos participantes realizou qualquer tipo de exercício físico nas 48 horas que antecederam a coleta sanguínea. As coletas sanguíneas ocorreram nos dois momentos (pré e pós) entre às 7:30 e 8:30 da manhã. Após a coleta, a amostra de sangue foi mantida em temperatura ambiente de $20-25^{\circ} \mathrm{C}$ por 45 minutos e centrifugada por 10 minutos a $1.500 \mathrm{rpm}$ (Megafuge 1.0R, Heraeus, Langenselbold, Alemanha). O soro foi então removido e mantido a $25^{\circ} \mathrm{C}$ para análise posterior. As concentraçốes séricas de cortisol foram determinadas usando kits de radioimunoensaio (Cortisol Coat-Count-RIA; DPC Medlab, Los Angeles, CA, EUA). Todos os ensaios foram realizados de acordo com as instruções dos fabricantes por técnicos de laboratório licenciados e certificados.

Vinte e quatro horas após a primeira avaliação, os participantes realizaram uma sessão de treino de força em circuito (10 exercícios) que consistiu em uma única sé-

\section{Tabela 1. Caracterização da amostra ( $n=10)$.}

MÉDIA

Idade

Peso (kg)

Estatura (m)

$\mathrm{IMC}^{1}$

$\mathrm{RCQ}^{2}$

Gordura $(\%)^{3}$

Massa magro (kg)

PAS (mmHg) 5

$\operatorname{PAD}(\mathrm{mmHg})^{6}$

Estresse $^{7}$ rie de 12 repetições (cada exercício) com intensidade 17-18 na escala de Borg de 20 pontos. Antes do início do treino de força os voluntários realizaram um aquecimento escala de Borg. O circuito de treinamento de força foi composto pelos seguintes exercícios: supino reto com barra, remada sentada, rosca bíceps, tríceps no cross, agachamento na barra guiada, voador, puxada frente na polia alta, rosca scott, tríceps na máquina e leg press $45^{\circ}$. A transição entre cada exercício foi de 15 a 20 segundos. Vinte e quatro horas após a sessão de treino todos os procedimentos e coletas realizados na primeira avaliação foram repetidos seguindo rigorosamente os mesmos padrões.

Após a tabulação, os dados foram analisados estatisticamente. Os dados foram apresentados em média e desvio padrão. Para testar a normalidade foi aplicado o teste de Shapiro-Wilk. Para investigar as mudanças nas medidas do nível de cortisol e da VFC pré e pós sessão de treinamento foi utilizado a Anova de duas vias com pos hoc de Bonferroni. Foi aplicada a correlação de Pearson ( $\mathrm{r}$ ) entre as variáveis cortisol, score de estresse e os índices de VFC. O nível de significância adotado foi de 5\%. A análise do poder da amostra foi realizada a post hoc através do software G*Power $3.1 \mathrm{com}$ alpha de 0,05 . Os dados foram analisados utilizando o pacote estatístico IBM SPSS 21.0 e o GraphPad Prisma. e esteira rolante com intensidade de 15 na

1indice de Massa Corporal; ${ }^{2}$ Relação Cintura Quadril; ${ }^{3}$ Percentual de Gordura Corporal; ${ }^{4}$ Massa magra em quilos; ${ }^{5}$ Pressão Arterial Sistólica; ${ }^{6}$ Pressão Arterial Diastólica; ${ }^{7}$ Score da Escala de Estresse Percebido. Autoria local 2020. 
Tabela 2. Índices da VFC pré e pós treino de força $(n=10)$.

$$
\text { VFC }
$$

REPOUSO

MÉDIA $\pm \mathrm{DP}$

\section{SDNN (ms)}

$\operatorname{RMSSD}(\mathrm{ms})$

$71,23 \pm 26,38$

$47,70 \pm 26,60$

pNN50 (\%)

$24,28 \pm 21,87$

$\mathrm{HF}$ (ms)

$1047,86 \pm 1126,62$

LF (ms)

HF (nu)

$\mathrm{LF}$ (nu)

LF/HF (ms)

$\mathrm{SD} 1$ (ms)

$\mathrm{SD} 2$ (ms)

$\mathrm{SD} 1 / \mathrm{SD} 2$ (ms)
$1384,27 \pm 854,37$

$37,73 \pm 16,65$

$62,20 \pm 16,65$

$2,12 \pm 1,27$

$33,74 \pm 18,82$

$94,38 \pm 33,79$

$0,45 \pm 0,42$

\section{PÓS TREINO}

MÉDIA \pm DP

$\mathrm{P}$

$65,69 \pm 19,31$

0,97

$43,65 \pm 20,13$

0,36

$21,37 \pm 18,68$

0,42

$811,29 \pm 554,36$

0,64

$1191,67 \pm 579,65$

0,16

$37,82 \pm 11,46$

0,97

$62,12 \pm 11,46$

0,97

$1,90 \pm 0,97$

0,22

$30,87 \pm 14,24$

0,36

$87,33 \pm 24,39$

0,24

$0,38 \pm 0,10$
VFC: Variabilidade da Frequência Cardíaca; DP: Desvio Padrão; SDNN: Desvio padrão de todos os intervalos RR normais em um intervalo de tempo; RMSSD: Raiz quadrada da média do quadrado das diferenças entre os intervalos RR; pNN50: Porcentagem dos intervalos RR adjacentes com diferença de duração maior que 50 milissegundos; HF: Variação que corresponde a modulação respiratória; LF: Variação decorrente da atuação conjunta dos componentes simpático e parassimpático; LF/HF: alteração absoluta e relativa entre os componentes simpáticos e parassimpático. SD1: índice instantâneo da variação entre batimentos; SD2: Representa a VFC em registro de longo prazo; SD1/SD2: Razão entre as variações curta e longa dos intervalos RR. Autoria local 2020.

\section{Figura 2. Níveis de cortisol antes e pós-treinamento de força. Autoria local 2020.}

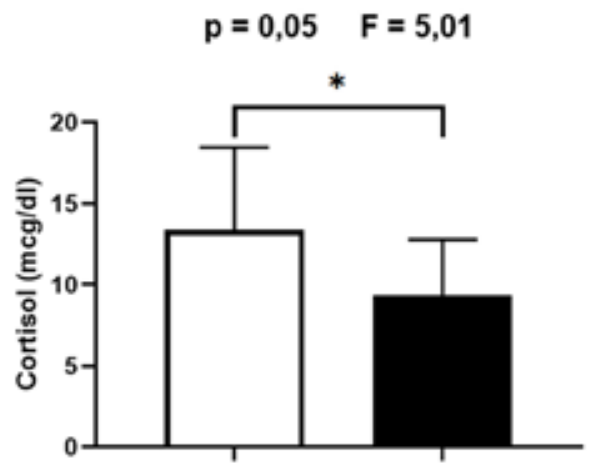

Repouso

Pós Treino

Figura 3. Correlações: A) Score de estresse e nível de cortisol; B) Cortisol e índice LFms. Autoria local 2020.
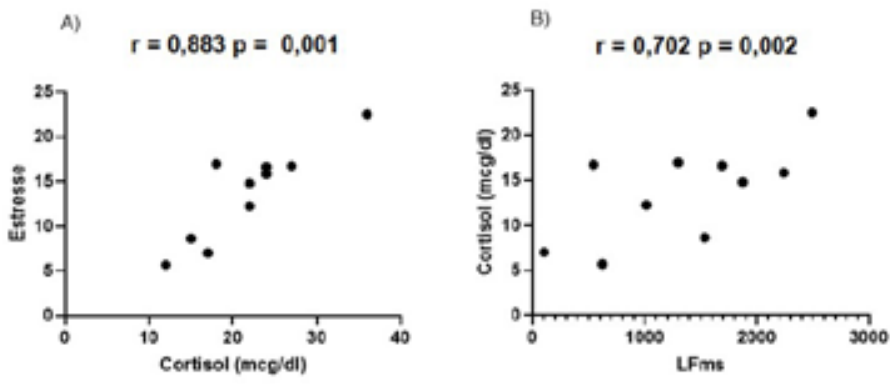

\section{RESULTADO}

Na tabela 1 estão apresentados os dados de caracterização da amostra. Para o cortisol o poder estatístico post hoc foi de 1.00 . O score médio dos voluntários no PSS foi $21,36 \pm 6,56$. Redução significativa foi observada nos níveis de cortisol um dia após a sessão de treinamento de força (figura 2).

Não foi observado nenhuma alteração estatisticamente significativa ( $p>0,05)$ nos índices da VFC no domínio do tempo e da frequência (tabela 2). A PAS e PAD também não apresentaram mudanças estatisticamente significativas $(p>0,05)$ após um dia do treinamento de força.

Uma forte correlação positiva foi observada entre o score de estresse e os níveis de cortisol bem como entre o cortisol e o índice LFms em repouso (figura 3), além de uma forte correlação positiva $(r=0,700 \mathrm{p}=0,024)$ entre a razão SD1/SD2 e a massa magro.

\section{DISCUSSÃO}

Este estudo teve por objetivo investigar a resposta de uma única sessão de treinamento de força nos níveis de cortisol e correlacionar com estresse percebido em homens adultos durante a pandemia da COVID-19. Até onde sabemos, este é o primeiro estudo a investigar o efeito do treino de força no estresse durante uma pandemia. Os principais achados deste estudo demostram que houve uma redução nos níveis de cortisol um dia após uma única sessão de treino de força realizado com intensidade entre 17-18 na Escala de Borg. Além disso, uma forte correlação foi observada entre o nível de cortisol em repouso com o escore de estresse percebido e o componente LFms, também foi observada uma correlação forte entre a massa magro e a razão $\mathrm{SD} 1 / \mathrm{SD} 2$ em repouso.

O cortisol é um hormônio corticosteroide da família dos esteroides. A produção ocorre no córtex superior da glândula suprarrenal. Tem como função estimular a quebra de proteínas, gorduras e a síntese de glicose no fígado. Tem alta relação com o estresse, pois ativa respostas fisiológicas em situação de emergência como aumentando 


\section{artigo}

a pressão arterial e disponibilidade de glicose que é utilizada como fonte de energia para a contração muscular ${ }^{17}$. Em nosso estudo, o cortisol apresentou uma forte correlação positiva $(\mathrm{r}=0,883, \mathrm{p}=0,001)$ com o score de estresse. Apesar do cortisol desencadear estas importantes alterações, simultaneamente implica na paralisação de todas as funções de recuperação e anabolismo. Este fato faz com que o cortisol desempenhe o papel influenciador no catabolismo muscular ${ }^{18,19}$. O estresse crônico pode acarretar uma constante elevação nos níveis de cortisol e influenciar na redução da massa muscular decorrente do catabolismo. A diminuição da massa muscular exerce forte influência no metabolismo, impactando na redução do gasto calórico basal, na queima de gordura e reduz a utilização da glicose, o que pode levar ao desenvolvimento de doenças crônico metabólicas ${ }^{20}$. Em nosso estudo uma única sessão de treinamento de força com intensidade 17 na escala de Borg se mostrou eficiente na redução significativa nos níveis de cortisol sanguíneo um dia após a realização do treino. Estudos apontam que o cortisol se mostra mais susceptível a aumentar seus níveis após exercícios com maiores volumes de treino ${ }^{21,22}$. A alta intensidade, porém com menores volumes parecem não exercer grande influência no aumento dos níveis de cortisol. No estudo de Geisler e seus colaboradores ${ }^{23}$ foi observado uma redução no cortisol após 45 minutos do treino de força realizado a $75 \%$ de 1 RM. O exercício de força com séries combinadas também demostrou reduções nos níveis de cortisol ${ }^{24}$. Os achados de Rosa e colaboradores ${ }^{25,26}$ também demostram redução significativa do cortisol após uma sessão de treino combinado (aeróbio e força). Em nosso estudo o treinamento de força realizado com apenas uma única série em circuito, provocou uma redução nos níveis de cortisol corroborando com estes estudos. Foi observado uma correlação forte entre os níveis de cortisol e o índice LFms em repouso. Este componente relaciona-se com a atividade simpática. $\mathrm{O}$ aumento dos valores do índice LFms reduz a VFC. Estudos observaram redução da VFC e dos níveis de cortisol pós sessão de meditação ${ }^{27}$ e antecipação em situação de estresse é a resposta do cortisol ${ }^{28}$. Nosso estudo mostrou uma associação positiva entre o cortisol e LFms em repouso inferindo uma maior atividade simpática em repouso. Não foi observado em nosso estudo alteração estatisticamente significativa nos índices da VFC pós treinamento de força. No estudo de Figueiredo e colaboradores ${ }^{29}$ foi observado uma maior magnitude no tônus simpática pós treinamento de força ( 5 series de 8-10 repetições) realizado com intensidade de $70 \%$ de 1 RM quando comparado ao grupo que realizou apenas 1 série de exercício. Assim a VFC pode ser mais sensível ao volume de treino, aumentado a atividade simpática pós exercício. Uma vez que nosso protocolo se caracterizou como treino de baixo volume e alta intensidade, este pode ter sido o motivo pelo qual não foi observado modificações estatisti- camente significativas nos parâmetros da VFC. Entretanto o massa magra em nosso estudo se correlacionou fortemente com a razão SD1/SD2 que representa a variação (curta e longa) dos intervalos RR. Indivíduos com maiores valores de massa magra apresentam uma maior VFC ${ }^{30}$. Ademais, vem sendo observado uma associação do aumento da adiposidade corporal com o desequilíbrio autonômico ${ }^{31}$. Também o desequilíbrio simpático-vagal tem intima relação com a sarcopenia, comprovando a importância da manutenção da massa muscular na saúde autonômica ${ }^{32,33}$. Estes achados corroboram com nosso estudo e enfatiza a importância da redução do cortisol e como consequência, os seus efeitos catabólicos sobretudo neste período de pandemia.

\section{CONCLUSÃO}

Uma única sessão de treino de força com alta intensidade (Borg 17) se mostrou eficaz na redução do cortisol em homens adultos que apresentaram níveis elevados de estresse percebido durante a pandemia da COVID-19. Quando o objetivo for a atenuação do estresse emocional, treinamento curtos de força parece ser uma estratégia rápida e viável.

Aconselhamos pesquisa posteriores com diferentes volumes e intensidade de treino além de outras faixas etárias e gênero que permitam futuramente extrapolar estes resultados para outros grupos populacionais.

\section{REFERÊNCIAS}

1. Esakandari $H$, Nabi-Afjadi M, Fakkari-Afjadi J, Farahmandian N, Miresmaeili S-M, Bahreini E. A comprehensive review of COVID-19 characteristics. Biological Procedures Online. 2020;22:1-10.

2. Kannan S, Ali PSS, Sheeza A, Hemalatha K. COVID-19 (Novel Coronavirus 2019)-recent trends. Eur Rev Med Pharmacol Sci. 2020;24(4):2006-11.

3. Carraturo F, Del Giudice C, Morelli M, Cerullo V, Libralato G,

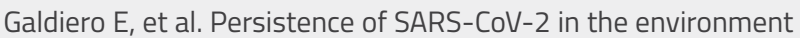
and COVID-19 transmission risk from environmental matrices and surfaces. Environmental Pollution. 2020:115010.
4. Sun P, Lu X, Xu C, Sun W, Pan B. Understanding of COVID 19 based on current evidence. Journal of medical virology. 2020;92(6):548-51.

5. Bohlken J, Schömig F, Lemke MR, Pumberger M, Riedel-Heller SG. COVID-19 Pandemic: Stress Experience of Healthcare Workers-A Short Current Review. Psychiatrische Praxis. 2020;47(4):190-7.

6. Ozamiz-Etxebarria N, Dosil-Santamaria M, Picaza-Gorrochategui M, Idoiaga-Mondragon N. Stress, anxiety, and depression levels in the initial stage of the COVID-19 outbreak in a population sample in the northern Spain. Cadernos de Saúde 


\section{REFERÊNCIAS}

Pública. 2020;36:e00054020.

7. Stanton R, To QG, Khalesi S, Williams SL, Alley SJ, Thwaite TL, et al. Depression, Anxiety and Stress during COVID-19: Associations with Changes in Physical Activity, Sleep, Tobacco and Alcohol Use in Australian Adults. International Journal of Environmental Research and Public Health. 2020;17(11):4065.

8. Narici M, De Vito G, Franchi M, Paoli A, Moro T, Marcolin G, et al. Impact of sedentarism due to the COVID-19 home confinement on neuromuscular, cardiovascular and metabolic health: Physiological and pathophysiological implications and recommendations for physical and nutritional countermeasures. European Journal of Sport Science. 2020:1-22.

9. Ranasinghe C, Ozemek C, Arena R. Exercise and well-being during COVID 19-time to boost your immunity. Expert Review of Anti-infective Therapy. 2020:1-6.

10. Chandrasekaran B, Ganesan TB. Sedentarism and chronic disease risk in COVID 19 lockdown-a scoping review. Scottish Medical Journal. 2020:0036933020946336.

11. Füzéki E, Groneberg DA, Banzer W. Physical activity during COVID-19 induced lockdown: recommendations. Journal of Occupational Medicine and Toxicology. 2020;15(1):1-5.

12. Lila N, Sudakov S. Method for Studies of Orientation and Exploratory Behavior in Humans. Effects of Emotional Stress. Bulletin of experimental biology and medicine. 2018;165(3):412-4.

13. Chowdhury R, Mukherjee A, Mitra K, Naskar S, Karmakar PR, Lahiri SK. Perceived psychological stress among undergraduate medical students: Role of academic factors. Indian Journal of Public Health. 2017;61(1):55.

14. Jackson AS, Pollock ML. Practical assessment of body composition. The Physician and Sportsmedicine. 1985;13(5):76-90.

15. Osayande $\mathrm{OE}$, Azekhumen GN, Obuzor EO. A comparative study of different body fat measuring instruments. Nigerian Journal of Physiological Sciences. 2018;33(2):125-8.

16. Pumprla J, Howorka K, Groves D, Chester M, Nolan J. Functional assessment of heart rate variability: physiological basis and practical applications. International journal of cardiology. 2002;84(1):1-14.

17. Perogamvros I, Ray DW, Trainer PJ. Regulation of cortisol bioavailability-effects on hormone measurement and action. Nature Reviews Endocrinology. 2012;8(12):717-27.

18. Popovic B, Popovic D, Macut D, Antic IB, Isailovic T, Ognjanovic $S$, et al. Acute response to endurance exercise stress: focus on catabolic/anabolic interplay between cortisol, testosterone, and sex hormone binding globulin in professional athletes. Journal of medical biochemistry. 2019;38(1):6-12.

19. Yanagita I, Fujihara Y, Kitajima Y, Tajima M, Honda M, Kawajiri T, et al. A high serum cortisol/DHEA-S ratio is a risk factor for sarcopenia in elderly diabetic patients. Journal of the Endocrine Society. 2019;3(4):801-13.

20. Yeung CHC, Yeung SLA, Fong SSM, Schooling CM. Lean mass, grip strength and risk of type 2 diabetes: a bi-directional Mende- lian randomisation study. Diabetologia. 2019;62(5):789-99.

21. Bartolomei S, Sadres E, Church DD, Arroyo E, Gordon III JA, Varanoske AN, et al. Comparison of the recovery response from high-intensity and high-volume resistance exercise in trained men. European journal of applied physiology. 2017;117(7):128798.

22. Kraemer WJ, Ratamess NA. Hormonal responses and adaptations to resistance exercise and training. Sports medicine. 2005;35(4):339-61.

23. Geisler S, Aussieker T, Paldauf S, Scholz S, Kurz M, Jungs S, et al. Salivary testosterone and cortisol concentrations after two different resistance training exercises. The Journal of sports medicine and physical fitness. 2019;59(6):1030-5.

24. Weakley JJ, Till K, Read DB, Roe GA, Darrall-Jones J, Phibbs $\mathrm{PJ}$, et al. The effects of traditional, superset, and tri-set resistance training structures on perceived intensity and physiological responses. European journal of applied physiology. 2017;117(9):1877-89.

25. Rosa G, Dantas E, Biehl C, e Silva HdC, Montano M, De Mello $\mathrm{D}$. Leptin, cortisol and distinct concurrent training sequences. International journal of sports medicine. 2012;33(03):177-80.

26. Rosa G, Dantas EH, de Mello DB. The response of serum leptin, cortisol and zinc concentrations to concurrent training. Hormones. 2011;10(3):215-21.

27. Blase KL, van Waning A. Heart rate variability, cortisol and attention focus during Shamatha quiescence meditation. Applied psychophysiology and biofeedback. 2019;44(4):331-42.

28. Pulopulos MM, Vanderhasselt M-A, De Raedt R. Association between changes in heart rate variability during the anticipation of a stressful situation and the stress-induced cortisol response. Psychoneuroendocrinology. 2018;94:63-71.

29. Figueiredo T, Rhea MR, Peterson M, Miranda $H$, Bentes CM, dos Reis VMdR, et al. Influence of number of sets on blood pressure and heart rate variability after a strength training session. 2015;29(6):1556-63.

30. Andrew ME, Shengqiao L, Wactawski Wende J, Dorn JP, Mnatsakanova A, Charles LE, et al. Adiposity, muscle, and physical activity: predictors of perturbations in heart rate variability. American Journal of Human Biology. 2013;25(3):370-7.

31. Triggiani Al, Valenzano A, Trimigno V, Di Palma A, Moscatelli F, Cibelli $\mathrm{G}$, et al. Heart rate variability reduction is related to a high amount of visceral adiposity in healthy young women. PloS one. 2019;14(9):e0223058.

32. de Freitas VP, da Silva Passos R, Oliveira AA, Ribeiro IJ, Freire IV, Schettino $L$, et al. Sarcopenia is associated to an impaired autonomic heart rate modulation in community-dwelling old adults. Archives of gerontology and geriatrics. 2018;76:120-4.

33. Fonseca GWPd, Santos MRd, Souza FRd, Costa MJAd, Haehling Sv, Takayama L, et al. Sympatho-vagal imbalance is associated with sarcopenia in male patients with heart failure. Arquivos brasileiros de cardiologia. 2019;112(6):739-46. 